\title{
Technical note: Three-dimensional imaging of rumen tissue for morphometric analysis using micro-computed tomography
}

\author{
M. A. Steele, ${ }^{\star 1}$ F. Garcia, † M. Lowerison, $\ddagger$ K. Gordon,§ J. A. Metcalf, ${ }^{*}$ and M. Hurtig† \\ *Nutreco Canada Agresearch, Guelph, Ontario, Canada N1G 4T2 \\ †Clinical Studies, Ontario Veterinary College, University of Guelph, Guelph, Ontario, Canada N1G 2W1 \\ ¥Clinical Research Unit, Faculty of Medicine, University of Calgary, Alberta, Canada T2N 1N4 \\ §School of Engineering, University of Guelph, Guelph, Ontario, Canada N1G 2W1
}

\begin{abstract}
Rumen development in calves has been evaluated by measuring papillae length, width, and density using microscopy for over $50 \mathrm{yr}$. Although common in the literature, disadvantages to this method exist, such as large variations in rumen papillae size and shape, small numbers of total papillae being measured, and the time required. The objective of this study was to develop a more effective technique for assessing rumen papillae using micro-computed tomography (micro-CT) and to compare this technique with microscopy. Rumen tissue was collected from the ventral sac of 20 postweaned bull calves at $55 \mathrm{~d}$ of age, immediately fixed in $10 \%$ neutral buffered formalin for $48 \mathrm{~h}$, and stored in $70 \%$ ethanol at $4^{\circ} \mathrm{C}$ before the contrast enhancement. After evaluation of contrast-enhancement protocols, it was determined that mercury chloride provided the most pronounced contrast for accurate micro-CT imaging based on relative density of the papillae. A $1-\mathrm{cm}^{2}$ tissue section from the ventral sac of all bull calves was tensioned on a rapid prototyped curved plastic holder and imaged at $45 \mu \mathrm{m}$ resolution for $56 \mathrm{~min}$ using a GE Locus Explore micro-CT (General Electric, Milwaukee, WI). MicroView V2.2 software (General Electric) was used to create a 3 -dimensional virtual model of the entire sample. The length and width of papillae were measured 3-dimensionally and compared with measurements of papillae under the light microscope taken from the same region. The length and width measurements using micro-CT $(2.47 \pm 0.12$ and $0.55 \pm 0.01 \mathrm{~mm})$ compared with light microscope $(2.96 \pm 0.03$ and $0.86 \pm$ $0.01 \mathrm{~mm}$ ) were significantly smaller. The difference may reflect a more accurate determination in the base of the rumen tissue with micro-CT or the specificity of mercury chloride to bind only to intact rumen tissue. The mean number of papillae per centimeter squared viewed using micro-CT was $128.5 \pm 33.9$ with a total surface
\end{abstract}

Received May 17, 2014.

Accepted August 22, 2014.

${ }^{1}$ Corresponding author: masteele@ualberta.ca area of $681.8 \pm 112.4 \mathrm{~mm}^{2}$ and volume of $156 \mathrm{~mm}^{3}$ per sample. Micro-CT data demonstrated that surface area and volume are positively associated and that papillae length was negatively associated with papillae per centimeter squared and positively associated with total volume of tissue section. This study represents the first time that micro-CT has been being used to assess morphology of rumen tissue. Micro-CT has the potential to improve the accuracy and efficiency of rumen tissue measurements; however, more standardization of each factor involved in tissue preparation, imaging, and location of papillae measurements is required.

Key words: development, morphology, micro-computed tomography

\section{Technical Note}

The ruminant forestomach is primarily known for its role in microbial fermentation, although it also plays a major role in host defense, nutrient absorption, and metabolism (Gäbel et al., 2002). Thousands of fingerlike projections called papillae line the ruminant forestomach and function to increase the surface area of absorption of the end products of ruminal fermentation (Steele et al., 2011a). During the lifespan of the ruminant, the microarchitecture of the rumen papillae undergoes dramatic adaptations, especially during the transition of weaning. This development involves a combination of rapid cellular differentiation in the rumen lining resulting in increased rumen papillae size, thus more surface area for the absorption of ruminal fermentation end products (Baldwin et al., 2004). Inadequate rumen papillae development and dry feed intake before and after weaning is often associated with decreased weight gain, performance, and welfare (Khan et al., 2011). Taking this into consideration, identifying feeding regimens and functional nutrients that encourage rumen papillae growth and maturation may improve production performance, as well as the health and welfare of the young ruminant during the transition of weaning. 
The development and adaptations of the rumen is commonly assessed by rumen mass (Baldwin et al., 2004) and relative gene expression techniques (Penner et al., 2011; Steele et al., 2011a,b); however, the gold standard lies in assessing rumen papillae length, width, and density using microscopy (Lesmeister et al., 2004). Two-dimensional microscopic measurements are an indirect method of assessing the surface area for absorption and have been employed in ruminant physiology and nutrition for over 50 yr. The rumen consists of multiple compartments (caudal dorsal blind sac, cranial dorsal sac, cranial ventral sac, and the caudal and ventral portions of the ventral and dorsal blind sac), but most studies evaluating rumen papillae morphology focus only on specific sites, namely the ventral or dorsal sac where papillae are most developed (Lesmeister et al., 2004; Benschop and Cant, 2009). Furthermore, due to the time-consuming nature of the methodology, generally less than 20 papillae length and width measurements per site are measured. Large variations in papillae measurements between studies exist, which may reflect the diverse treatments or the timing of sampling (Lesmeister et al., 2004; Hill et al., 2005; Gorka et al., 2009; Roth et al., 2009). Nevertheless, most studies do not provide a detailed description of their methodology which may contribute to the discrepancies in the scientific literature.

Perhaps the largest source of error in rumen papillae measurements comes from the inherent complexity of ruminal morphological changes during weaning - a process that remains largely undescribed. For example, the surface of the rumen during weaning displays contours not evident in mature ruminants (Figure 1a). Crosssections of these contours examined under the light microscope (Figure 1b) show that the rumen epithelium at weaning displays a polyp-like structure where several immature papillae of different sizes and shapes protrude. This observation requires more investigation, as it likely contributes to the inconsistencies in the literature and, more importantly, could be an important event in rumen structural development. Furthermore, a closer look at rumen papillae after weaning under a stereoscope displays a large variation in rumen papillae size in adjacent papillae in the same section of rumen (Figure 1a). These 2 factors, rarely discussed in the literature, create significant challenges when collecting, analyzing, and interpreting papillae length and width measurements in ruminants during weaning.

A technical approach that may address the accuracy and time limitations of microscopic measurements of rumen papillae is to visualize rumen tissue in 3 dimensions using micro-computed tomography (micro-CT). $\mathrm{X}$-ray tomography has been used extensively in medical research to create cross sections of 3-dimensional objects that can later be used to recreate a virtual model without destroying the original sample. Over the last decade, the field of x-ray tomography has been revolutionized through technical developments which allow for pixel sizes of the cross-section to be viewed in the micrometer range (Metscher, 2009). Micro-CT has proven to be a valuable technique for the imaging of bone structures; however, the visualization of soft tissue is still a challenge due to low $\mathrm{x}$-ray contrast (Pauwels et al., 2013). By finding the optimal contrast enhancement it may be possible for multiple rumen tissue samples to be scanned simultaneously using a micro-CT to create 3-dimensional models of large tissues. These large sections could consist of thousands of rumen papillae which can be instantaneously measured for surface area. It further enables the technician to measure the length and width of individual rumen papillae in a 3-dimensional manner, improving the accuracy of measurements. In spite of its potential to the field of gastrointestinal research, a technique has not been developed to use micro-CT to measure gastrointestinal morphology. Therefore, the objective of our study was to develop a protocol for the measurement of rumen papillae length, width, and surface area using micro-CT and compare the results to the classical microscopic technique.

To meet this objective, the rumen tissue from the ventral sac of 20 bull calves was harvested on d 55 of life ( $7 \mathrm{~d}$ postweaning). All animal procedures for raising and euthanizing the bulls were approved by the $\mathrm{Nu}-$ treco Canada Agresearch Animal Care Committee in accordance with the Canadian Council on Animal Care (1993) guidelines. Calves were slaughtered by captive bolt pistol and exsanguination and the rumen was immediately harvested, rinsed in tap water, and washed in PBS. A $3-\mathrm{cm}^{2}$ section of tissue was dissected from the ventral sac of the rumen and pinned to dental wax (Canemco Inc., Gore, QC), to prevent tissue folding, and submersed in neutral buffered formalin (10\%) for $48 \mathrm{~h}$ at a volume of over 10:1. After $48 \mathrm{~h}$ of fixation the rumen tissue was transferred to a solution of $70 \%$ ethanol at a volume of $10: 1$ and stored at $4^{\circ} \mathrm{C}$ until staining for micro-CT.

To optimize the accuracy of the micro-CT imaging, a series of staining protocols were tested to determine the optimal contrast enhancement specific for rumen tissue. Tissue submerged in $70 \%$ ethanol was removed from the fridge, trimmed to an area of $1 \mathrm{~cm}^{2}$ using a microtome blade, and mounted on a rapid prototype curved plastic holder (Figure 2). Plastic was used for the specimen holder to eliminate the chance of x-ray dispersion and provided a sealed humidified environment. The holder was designed using Solidworks software (Dassault Systemes, Waltham, MA) with the predetermined 

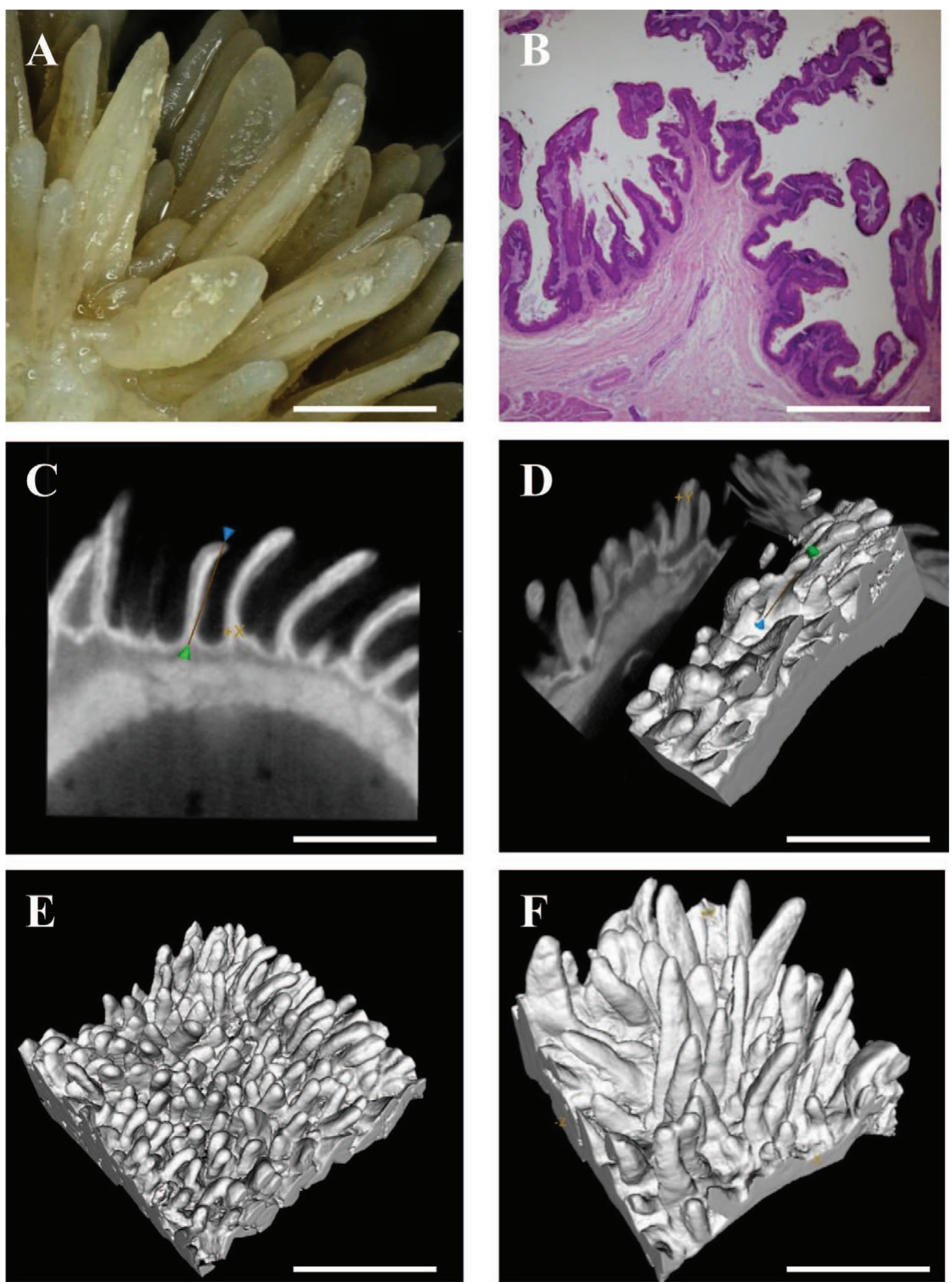

Figure 1. Images of the ruminal surface in calves during weaning under the light microscope or stereoscope. (a) Stereoscope image of rumen papillae size variation within each region $($ bar $=200 \mu \mathrm{m})$. (b) Light micrograph of polyp-like structure with papillae protruding $($ bar $=200$ $\mu \mathrm{m})$. (c) Cross-section of rumen tissue under the micro-computed tomography (micro-CT) equipment with mercury chloride stain showcasing the preferential penetration of the epithelium $(\mathrm{bar}=200 \mu \mathrm{m})$. (d) Three-dimensional reconstruction of rumen papillae cross-section to facilitate papillae in measurements under the micro-CT (bar $=200 \mu \mathrm{m}$ ). (e) Three-dimensional isosurface reconstruction of rumen tissue under the microCT with smaller but more dense papillae (bar $=200 \mu \mathrm{m})$. (f) Three-dimensional isosurface reconstruction of rumen tissue under the micro-CT with longer, but less dense papillae (bar $=200 \mu \mathrm{m})$. Color version available in the online PDF. 

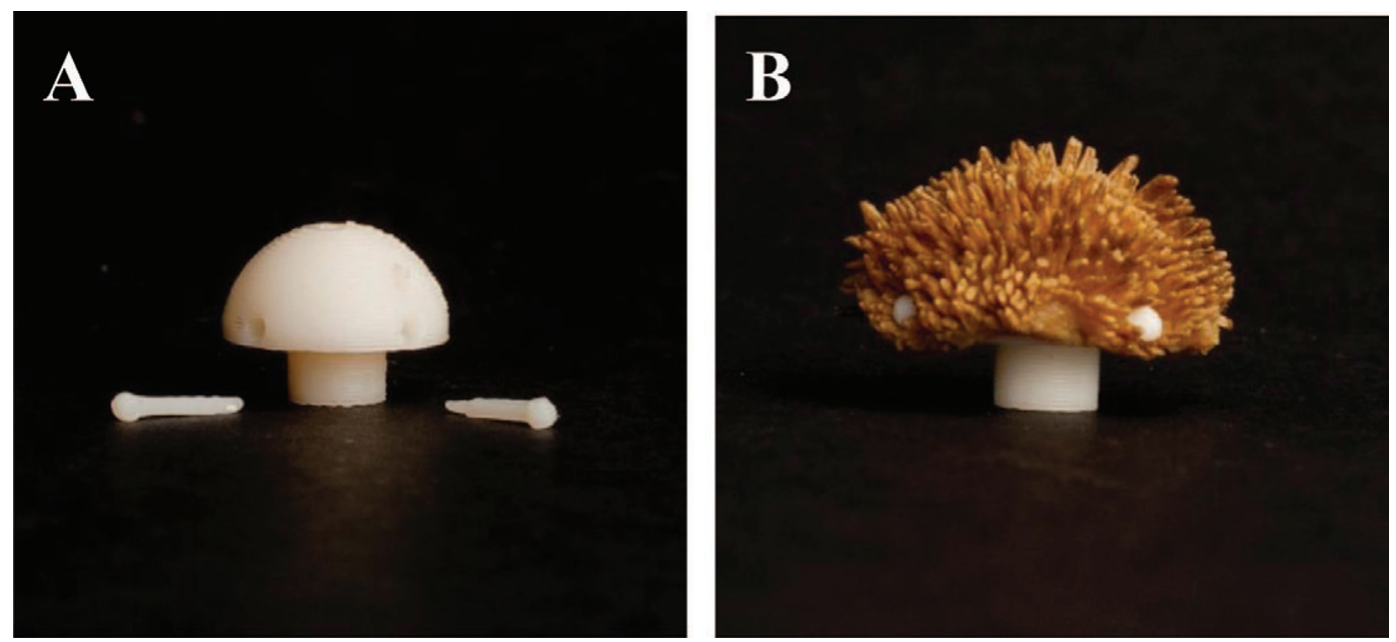

Figure 2. Photographic images of plastic holder and pegs (a) before mounting ruminal tissue, and (b) after mounting tissue. Color version available in the online PDF.

dimensions consisting of a circular base with a diameter of $8 \mathrm{~mm}$ with a curved top to the highest point of 8 $\mathrm{mm}$. The rumen tissue was mounted on plastic that was curved for 2 critical reasons. First, to minimize papillae touching, which increases the penetration of the stain to the base of the papillae, thereby enhancing the contrast of the individual papillae; second, if papillae are crowded together by the elastic tissue contraction in the rumen wall the base of the papillae will be touching, making it impossible visualize the base of the papillae. This would lead to an underestimation of papillae length and overall surface area.

Common stains specifically used for soft tissue contrast enhancement to improve micro-CT visualization were evaluated as described by Pauwels et al. (2013) and Metscher (2009). In brief, the stains tested included phosphomolybdic acid $(0.27 \mathrm{~mol} / \mathrm{L})$, phosphotungstic acid $(0.17 \mathrm{~mol} / \mathrm{L})$, ammonium molybdate $(0.26 \mathrm{~mol} / \mathrm{L})$, and mercury chloride $(0.55 \mathrm{~mol} / \mathrm{L})$. All rumen tissues were initially incubated for $24 \mathrm{~h}$ using the stains diluted in distilled water $(20 \mathrm{~mL}$ each). After incubation with stains, the rumen tissue was scanned in air within a humidified chamber at $45 \mu \mathrm{m}$ resolution for $56 \mathrm{~min}$ using a GE Locus Explore micro-CT (General Electric, Milwaukee, WI). The GE MicroView V2.2 software (General Electric) was used to create a 3-dimensional virtual model of the entire sample and contrast numbers were compared.

Contrast numbers were calculated by dividing the difference between the highest gray value and the lowest gray value by the highest gray value shown on the histogram (Pauwels et al., 2013). The contrast numbers for phosphomolybdic acid (0.66), ammonium molybdate (0.86), and mercury chloride (0.60) were all high in comparison to previously reported values from soft tissue in the literature (Pauwels et al., 2013). Phosphotungstic acid displayed the lowest contrast number (0.36) and was consequently discarded from the protocol.

The image threshold value was also used as a parameter for the selection of the stain. Very high greyscale values above 10,000 interfere with the calculation of the micro-CT image parameters. In preliminary tests, mercury chloride and phosphotungstic acid created images with an ideal dynamic range (Pauwels et al., 2013); although ammonium molybdate created higher contrast, it was discounted due to rapid precipitation and instability when combined with tissue samples. Further investigation of the stains, mercury chloride and phosphomolybdic acid, showed that decreasing the time of staining from 24 to $2 \mathrm{~h}$ yielded equivalent contrast enhancement for mercury chloride, but not for phosphomolybdic acid. Furthermore, the mercury chloride preferentially stained the living epithelial layers of the rumen tissue (stratum basale, spinosum, and granulosum), but not the outermost cornified layer or the connective and muscular tissue below the papillae (Figure 1c). The preferential staining of the living strata of the epithelium, as well as the speed of penetration, offered advantages which led us to select mercury chloride staining over phosphomolybidic acid.

Once it was determined that mercury chloride staining was the most robust for rumen tissue application, a $1.5-\mathrm{cm}^{2}$ tissue section from the ventral sac of all 20 bull calves was dissected and mounted on the plastic holders. After staining with mercury chloride for $2 \mathrm{~h}, 4$ samples were scanned simultaneously at $45 \mu \mathrm{m}$ resolution. MicroView V2.2 software created a 3-dimensional 
model of all samples, and a $1-\mathrm{cm}^{3}$ region of interest for each sample was defined and saved electronically before analysis. Rumen papillae length and width was measured in 4 cross-sections per $1-\mathrm{cm}^{2}$ sample, $250 \mu \mathrm{m}$ apart from each other. For each defined cross-section, a 3-dimensional region of interest consisting of 250 $\mu \mathrm{m}$ thickness was constructed using Microview V2.2 software (Figure 1d). From this construction, length and width of approximately 10 rumen papillae were measured 3 dimensionally. The base of each papillae was determined by delineating epithelium at the floor of the papillae. The length was derived from measuring the base from the middle of the papillae to the tip. Also, the width was measured at the exact midpoint of the length measurement for all papillae.

Surface area and volumes can be measured automatically using Microview 2.2 in a defined region of interest. To create a region of interest for rumen papillae surface area and volume assessment, the connective and muscular tissue below the papillae was eliminated from the region of interest. This was accomplished by visually identifying the base of all of the papillae in the sample in planes of $50 \mu \mathrm{m}$ for a total of 20 planes per $1-\mathrm{cm}^{2}$ tissue section. The regions below the base of the papillae could be deleted from the initial region of interest to determine the volume and surface area of the papillae that would be exposed to the rumen milieu. Samples of the 3-dimensional reconstruction of rumen tissue from calves with small and large papillae are shown in Figure $1 \mathrm{e}$ and $1 \mathrm{f}$.

To compare micro-CT measurements of length and width to conventional 2-dimensional stereology measurements paired adjacent $1-\mathrm{cm}^{2}$ rumen tissue samples were collected. In brief, the $1-\mathrm{cm}^{2}$ tissue section was sliced into 4 equal sections of $250 \mu \mathrm{m}$ thickness using a microtome blade. Each section of tissue was examined and imaged under a stereomicroscope equipped with a video camera using $15 \times$ magnification Measurements of papillae length and width were recorded 2-dimensionally using Northern Eclipse Morphometry Software Version 5.15.4.0 (Empix Imaging, Mississauga, ON, Canada). Only intact rumen papillae not damaged by microtome slicing were measured in all 5 sections. The bottom of the papillae was defined by the dark line delineating the epithelium from the connective tissue at the base of the papillae.

Comparisons between micro-CT and stereology rumen papillae length and width measurements were made using a mixed procedure in $\mathrm{R}(\mathrm{R}$ Development Core Team, 2013). The length and width measurements using micro-CT $(2.47 \pm 0.12$ and $0.55 \pm 0.01 \mathrm{~mm})$ compared with stereoscopy $(2.96 \pm 0.03$ and $0.86 \pm$ $0.01 \mathrm{~mm})$ were significantly smaller $(P<0.0001)$. This difference may reflect a more accurate determination in the base of the rumen tissue with micro-CT from mercury chloride staining. Furthermore, the stereology measurements account for all layers of the epithelium, including the dead keratinocytes comprising the stratum corneum. In micro-CT samples it was also observed that the mercury chloride contrast only penetrated the live stratum basale, spinosum, and granulosum located beneath the corneum. The rumen epithelium is coated with a corneum that ranges from 1 to 10 cells and each cell can range from 5 to $25 \mu \mathrm{m}$ thickness and may be one explanation for the difference. Although micro-CT provides surface area of living strata, the importance of the corneum to ruminal health must be considered in all studies investigating the rumen epithelium. Therefore, attention to corneum thickness by other techniques such as light and electron microscopy in conjunction with micro-CT is recommended.

The fundamental advantage of micro-CT methodology compared with 2-dimensional stereology is that the surface area of hundreds of papillae can be calculated automatically using an isosurface calculation. The mean total surface area was $681.8 \pm 112.4 \mathrm{~mm}^{2}$ and volume of $156 \pm 98.4 \mathrm{~mm}^{3}$ for all virtually dissected samples, excluding connective and muscular tissue. The mean number of papillae in every micro-CT $1-\mathrm{cm}^{3}$ region of interest was $128.5 \pm 33.9$ in each sample as counted manually by a technician. Pearson correlations between all micro-CT measurements were calculated. The data showed that surface area and volume were positively associated $(P=0.04)$ and that papillae length was negatively associated $(P<0.001)$ with papillae per centimeter squared and positively associated $(P=0.02)$ with total volume of tissue section. No correlations $(P$ $>0.05)$ between rumen papillae length and width measurements were noted. Interestingly, no correlation $(P$ $>0.05)$ between length and surface area was observed in the data set, which suggests that having longer papillae does not necessarily mean a greater surface area. This finding challenges current dogma in most studies that interpret increased length of papillae with more surface area for nutrient absorption, which warrants further investigation.

Ultimately, our study proved successful in developing a protocol for the 3-dimensional visualization of rumen tissue and comparing the effectiveness of this technique against classical stereological methodology. The major advantage of the micro-CT technique is the efficient manner in which thousands of rumen papillae can be digitally reconstructed 3 dimensionally to generate results within hours of sample collection. Threedimensional analyses also offer a true determination of surface area without extrapolation of rumen papillae length, width, and density measurements. Moreover, linear measurements of papillae length and width can 
still be made in using micro- $\mathrm{CT}$, if required by the researcher. Such 3-dimensional measurement could inevitably offer a more accurate representation of complex biological material than its 2-dimensional counterpart.

One disadvantage of this new methodology is the inaccessibility that laboratories have to a micro-CT and the associated computing and software capabilities due to the large investment (approximately $\$ 80,000-120,000$ ). Yet, micro-CT technology is becoming more common and renting time (approximately $\$ 50-100$ per hour) offers opportunities for all laboratories to efficiently use. The micro-CT system used in this technical note is optimized for large samples with a resolution of 45 microns, which takes less than $1 \mathrm{~h}$ to scan 4 to 5 samples for a total cost of approximately $\$ 100$. Furthermore, the toxicity of contrast enhancement stains requires safety considerations, namely utilizing heavy metals for contrast enhancement, which may not be feasible in some labs. With access to a fume hood and proper residue handling procedures for hazardous waste, working with heavy metal contrasts can be easily accomplished.

In summary, micro-CT represents a technical advancement in our ability to examine the morphological structure of rumen papillae and potentially gastrointestinal tissue. The results from our study show that it is possible visualize rumen tissue using micro-CT when using the optimal contrast-enhancement stain. The robustness of the micro-CT technique requires more investigation, as the results from our study show advantages in the quickness of generating results, the number of papillae that can be measured, and a true 3 -dimensional quantification of surface area rather than an extrapolation of 2-dimensional measurements. Furthermore, the potential to distinguish between different tissue and strata types in rumen tissue is intriguing and may be very useful for answering future research questions related to epithelial structure and development. In addition, more sites of the rumen need to be evaluated and investigating the entire rumen in one scan would be optimal. Strong potential to use this technique to study mature dairy cow morphology of digestive tissues with repeated measures also exists if tissue can be biopsied. The potential of micro-CT to improve the accuracy and efficiency of rumen and gastrointestinal morphological structure evaluation and analysis warrant further investigations.

\section{ACKNOWLEDGMENTS}

We acknowledge the support of the Natural Sciences and Engineering Research Council of Canada (Ottawa,
ON, Canada). We also appreciate the technical assistance provided by Michelle Carson, Stephen Elgersma, Carolyn Innes (Nutreco Canada Agresearch), Andrew Hunt (Shur-Gain USA, Strykersville, NY), Kaela Shea, and John Phillips (School of Engineering, University of Guelph). Furthermore, the photographic expertise of Derek Sheflin (Pony Photography, Guelph, ON, Canada) was greatly appreciated.

\section{REFERENCES}

Baldwin, R. L., K. R. McLeod, J. L. Klotz, and R. N. Heitmann. 2004. Rumen development, intestinal growth and hepatic metabolism in the pre- and postweaning ruminant. J. Dairy Sci. 87:55-65.

Benschop, D. L., and J. P. Cant. 2009. Developmental changes in clearance of intravenous doses of glucose, acetate and $\beta$-hydroxybutyrate from plasma of calves. Livest. Sci. 122:177-185.

Canadian Council on Animal Care. 1993. Guide to the Care and Use of Experimental Animals. Canadian Council on Animal Care, Ottawa, ON, Canada.

Gäbel, G., J. R. Aschenbach, and F. Müller. 2002. Transfer of energy substrates across the ruminal epithelium: Implications and limitations. Anim. Health Res. Rev. 3:15-30.

Gorka, P., Z. M. Kowalski, P. Pietrzak, A. Kotunia, R. Kiljanczyk, J. Flaga, J. J. Holst, P. Guilloteau, and R. Zabielski. 2009. Effect of sodium butyrate supplementation in milk replacer and starter diet on rumen development in calves. Pol. J. Physiol. Pharmacol. 60:47-53.

Hill, S. R., B. A. Hopkins, S. Davidson, S. M. Bolt, D. E. Diaz, C. Brownie, T. Brown, G. B. Huntington, and L. W. Whitlow. 2005. Technical Note: Technique for dissection and analysis of the rumen in young calves. J. Dairy Sci. 88:324-326.

Khan, M. A., D. M. Weary, and M. A. G. Von Keyserlinkgk. 2011. Invited Review: Effects of milk ration on solid feed intake, weaning, and performance in dairy heifers. J. Dairy Sci. 94:1071-1081.

Lesmeister, K. E., P. R. Tozer, and A. J. Heinrichs. 2004. Development and analysis of a rumen tissue sampling procedure. J. Dairy Sci. 87:1336-1344.

Metscher, B. D. 2009. MicroCT for comparative morphology: Simple staining methods allow high contrast 3D imaging of diverse nonmineralized animal tissues. BMC Physiol. 9:11.

Pauwels, E., D. Van Loo, P. Cornillie, L. Brabant, and L. Van Hoorebeke. 2013. An exploratory study of contrast agents for soft tissue visualization by means of high resolution X-ray computed tomography imaging. J. Microsc. 250:21-31.

Penner, G. B., M. A. Steele, J. R. Aschenbach, and B. W. McBride. 2011. Molecular adaptation of ruminal epithelia to highly fermentable diets. J. Anim. Sci. 89:1108-1119.

R Development Core Team. 2013. R: A Language and Environment for Statistical Computing. R Foundation for Statistical Computing, Vienna, Austria.

Roth, B. A., N. M. Keil, L. Gygax, and E. Hillmann. 2009. Influence of weaning method on health status and rumen development in dairy calves. J. Dairy Sci. 92:645-656.

Steele, M. A., J. Croom, M. Kahler, O. AlZahal, S. E. Hook, K. Plaizier, and B. W. McBride. 2011a. Bovine rumen epithelium undergoes dramatic structural adaptations during grain-induced ruminal acidosis epithelial adaptation. Am. J. Physiol. Integr. Comp. Phys. 300:R1515-R1523.

Steele, M. A., G. Vandervoort, O. AlZahal, S. E. Hook, J. C. Matthews, and B. W. McBride. 2011b. Rumen epithelial adaptation to high-grain diets involves the coordinated regulation of genes involved in cholesterol homeostasis. Physiol. Genomics 43:308-316. 